\title{
Evolución de la capacidad funcional en adultos mayores hospitalizados en la unidad geriátrica de agudos del Hospital Clínico de la Universidad de Chile
}

\author{
VÍCTOR CARES L.a,b, CARLOS DOMÍNGUEZ C. ${ }^{a}$, \\ JAVIER FERNÁNDEZ M. ${ }^{a}$, RODRIGO FARÍAS C. ${ }^{a}$, WIN-TIN CHANG G. ${ }^{a}$, \\ GERARDO FASCE ${ }^{1}$, VÍCTOR CARRASCO ${ }^{1}$
}

\section{Evolution of functional capacity of older people during hospital stay}

Background: Up to 70\% of hospitalized older people experience a deterioration of their functional capacity during the course of hospital stay. This change has a dismal effect of quality of life and prognosis. Aim: To assess the change in functional status of older people during the course of hospitalization in a geriatric unit. Material and Methods: Review of medical records of 83 patients with a mean age of 79 years (70\% women), hospitalized in an acute geriatric unit of a clinical hospital, between 2007 and 2009. Functional capacity was assessed using Barthel and Lawthon scales in a basal period, on admission and on discharge. Results: Mean hospital stay was nine days. Median scores of Barthel scale on the basal period, on admission and on discharge were 90, 50 and 80, respectively. The figures for Lawthon scale were 4, 2 and 3 respectively. Seventy eight percent of patients lost functional capacity during hospital stay and $72 \%$ recovered their functional status on discharge. Conclusions: A great proportion of older people experience a loss of functional capacity during hospitalization. This deterioration can be reverted with an adequate geriatric management.

(Rev Med Chile 2013; 141: 419-427).

Key words: Frail elderly; Geriatrics; Hospitalization.

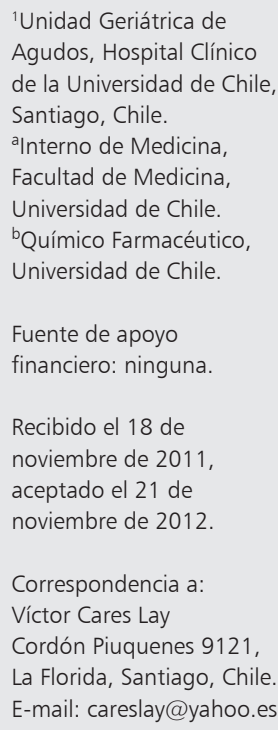

$\mathrm{E}$ n América Latina la prevalencia de adultos mayores (AM) de 60 o más años fue de $8 \%$ al año 2000 y se estima que para el año 2025 esta cifra ascenderá a 14,1\% ${ }^{1}$. En Chile las estadísticas indican algo muy similar, siendo 1 de cada 10 sujetos AM y para el año 2025 esta relación se estima en 1 de cada 5 personas ${ }^{2}$.

El enfoque de la salud en los AM es distinta al resto de la población, debido a la forma de presentación de las enfermedades, las múltiples patologías asociadas, la polifarmacia y su alteración funcional en el diario vivir, haciéndolos más susceptibles a la dependencia de otras personas y a la hospitalización, la cual se ha asociado a un mayor riesgo de institucionalización, delirium, depresión, morbimortalidad y deterioro funcional ${ }^{3}$.
Se ha descrito que $47 \%$ de los pacientes mayores de 60 años pueden ser considerados de alto riesgo para presentar deterioro funcional durante la hospitalización ${ }^{4}$ y que en otras series se reporta hasta $70 \%$ de deterioro funcional al momento de la hospitalización ${ }^{5}$, relacionándose con: el diagnóstico de ingreso, deterioro cognitivo previo, bajo nivel de actividad social, edad ${ }^{6} \mathrm{y}$ factores intrahospitalarios como el reposo prolongado, larga estancia y rehabilitación tardía ${ }^{5,7,8}$, todo lo cual es fundamental en la rehabilitación posterior de los pacientes, siendo menos auspiciosa en la medida que es mayor su deterioro funcional. Por lo tanto, es de vital importancia un diagnóstico precoz, un manejo interdisciplinario con una estadía en unidades especializadas para ayudar a la pronta 
restitución del paciente a la normalidad, evitando posibles complicaciones asociadas a la hospitalización. Si bien existen publicaciones en otros países que describen la evolución de la capacidad funcional de los pacientes geriátricos ${ }^{9,10}$, en nuestro país no hay reportes que aborden el tema, existiendo escasas unidades especializadas en la atención de pacientes geriátricos agudos.

El objetivo de este estudio es describir el perfil y la evolución de la capacidad funcional de AM de 65 o más años hospitalizados en la Unidad Geriátrica de Agudos (UGA) del Hospital Clínico de la Universidad de Chile (HCUCH).

\section{Materiales y Métodos}

El equipo interdisciplinario de la UGA está compuesto por geriatras, enfermera, psicóloga, farmacéutico clínico y auxiliares, además tiene el apoyo de kinesiólogo y terapeuta ocupacional, pertenecientes a la unidad de medicina física y de una asistente social, propia del hospital. Posee 4 salas con 11 camas en total, las que cuentan con medios de orientación para sus pacientes como calendario y reloj, entre otros.

Se realizó un estudio retrospectivo mediante la revisión de fichas clínicas de los pacientes AM de 65 o más años hospitalizados en la UGA del HCUCH desde agosto de 2007 hasta agosto de 2009. Se excluyeron pacientes ingresados para realizar una prueba o procedimiento, puntaje 0 en test de Barthel $^{11}$, puntaje mayor a 8 en test Pfeiffer ${ }^{12}$ al ingreso de la hospitalización, aquellos que presentaron demencia severa con afasia global, estado de coma o agonía, procedentes de otros servicios con estancia hospitalaria mayor a $72 \mathrm{~h}$ y fallecidos durante la hospitalización.

Se registraron la edad, sexo, diagnóstico de ingreso, comorbilidades, días de hospitalización, uso de fármacos, manejo hospitalario, capacidad funcional y presencia de delirium. La comorbilidad se evaluó mediante el índice de Charlson (ICh) (Anexo 1), el cual determina un valor pronóstico de fallecer al año en personas mayores de 65 años, al asignar un puntaje determinado a cada patología presentada $^{13}$.

El manejo hospitalario consideró el uso de sonda naso-gástrica, catéter urinario durante más de $48 \mathrm{~h}$ continuas, indicación médica de movilización, apoyo kinesiológico, psicológico y de terapeutas ocupacionales.
El estado funcional de las actividades básicas de la vida diaria (ABVD) y las actividades instrumentales de la vida diaria (AIVD) se evaluaron mediante el índice de Barthel (IB) ${ }^{11}$ (Anexo 2) y el índice de Lawton \& Brody (IL) ${ }^{14}$ (Anexo 3 ) respectivamente, los cuales determinan el grado de independencia en las áreas previamente mencionadas.

La detección de delirium se realizó mediante el algoritmo diagnóstico de $\mathrm{CAM}^{15}$ (Confusion Assessment Method) (Anexo 4), o con el diagnóstico registrado en la ficha médica.

El ICh se aplicó al momento del egreso hospitalario, el uso de fármacos y presencia de delirium se consideraron al ingreso, durante la hospitalización $y$ al egreso. Las ABVD y AIVD se evaluaron dos semanas previas a la hospitalización (estado basal), al ingreso y egreso hospitalario.

El presente trabajo contó con la aprobación del Comité Ético Científico para Investigación Biomédica en Seres Humanos del HCUCH, de la Sección de Geriatría, Departamento de Medicina del HCUCH. Se resguardó la confidencialidad y anonimato de los datos obtenidos, siendo estos utilizados únicamente para los objetivos ya descritos.

\section{Análisis estadístico}

Para variables continuas se determinó la normalidad mediante la prueba de Shapiro-Wilk. Presentándose como media y desviación estándar y analizándose mediante t-Student (edad y días de hospitalización). En el caso de variables discretas no paramétricas (índices de Barthel y de Lawton, Charlson, número de comorbilidades y fármacos) se presentan como mediana y rango intercuartílico analizándose mediante U de Mann-Whitney y Wilcoxon Signed-ranks test. Las variables cualitativas (sexo, comorbilidades, diagnósticos de ingreso, CAM y manejo hospitalario) se representan como porcentaje e intervalo de confianza, analizadas mediante $\chi^{2}$.

Se utilizó coeficiente de correlación de Spearman y regresión lineal simple para evaluar correlación entre variables dependiendo de tipo de distribución. Se consideró significativo un $\mathrm{p}<0,05$. El análisis estadístico se realizó con programa STATA $10^{\circledast}$ (StataCorpLP, Texas, USA).

\section{Resultados}

De un total de 378 pacientes hospitalizados durante el período de estudio, 83 cumplieron con los 
Tabla 1. Perfil de los pacientes hospitalizados en la UGA HCUCH

\begin{tabular}{|c|c|c|c|c|c|c|c|c|}
\hline \multirow[b]{2}{*}{ Pacientes $\left(\mathrm{N}^{\circ}\right)$} & \multirow[b]{2}{*}{83} & \multirow[t]{2}{*}{ Total } & \multicolumn{2}{|c|}{ Femenino } & \multicolumn{2}{|c|}{ Masculino } & \multicolumn{2}{|l|}{$\mathbf{p}$} \\
\hline & & & 58 & & 25 & & & \\
\hline Edad (Promedio años) & 79,4 & $\pm 6,8$ & 79,6 & $\pm 6,7$ & 78,8 & $\pm 7,1$ & 0,64 & $\nLeftarrow$ \\
\hline Comorbilidades $\left(X^{\circ}\right)$ & 3 & {$[2-4]$} & 4 & {$[2-5]$} & 3 & {$[2-4]$} & 0,07 & $\odot$ \\
\hline Índice Charlson ( $\left.X^{\circ}\right)$ & 2 & {$[1-3]$} & 1,5 & {$[1-2]$} & 2 & {$[1-4]$} & 0,18 & $\odot$ \\
\hline Fármacos Ingreso $\left(X^{\circ}\right)$ & 4 & {$[3-6]$} & 4 & {$[2-6]$} & 4 & {$[4-5]$} & 0,73 & $\odot$ \\
\hline Durante & 6 & {$[5-7]$} & 6 & {$[5-8]$} & 5,5 & {$[4-7]$} & 0,38 & $\odot$ \\
\hline Egreso & 5 & {$[3-7]$} & 5 & {$[4-7]$} & 5 & {$[3-7]$} & 0,47 & $\odot$ \\
\hline Diagnósticos de ingreso & & & & & & & & $\square$ \\
\hline$N A C^{*}$ & $22,9 \%$ & {$[14,4-33,4]$} & $22,4 \%$ & {$[12,5-35,3]$} & $24,0 \%$ & {$[9,4-45,1]$} & 0,88 & $\square$ \\
\hline ITU** & $6,0 \%$ & {$[1,9-13,5]$} & $5,5 \%$ & {$[1-14,3]$} & $8,0 \%$ & {$[0,9-26]$} & 0,64 & $\square$ \\
\hline Demencia & $4,8 \%$ & {$[1,3-11,9]$} & $6,9 \%$ & {$[1,9-16,7]$} & $0,0 \%$ & & 0,31 & $\square$ \\
\hline Bronquitis aguda & $3,6 \%$ & {$[0,75-10,2]$} & $5,2 \%$ & {$[1-14,3]$} & $0,0 \%$ & & 0,55 & $\square$ \\
\hline DM II*** descompensada & $3,6 \%$ & {$[0,75-10,2]$} & $1,7 \%$ & {$[0-9,2]$} & $8,0 \%$ & {$[0,9-26]$} & 0,21 & $\square$ \\
\hline Dolor torácico & $3,6 \%$ & {$[0,75-10,2]$} & $5,2 \%$ & {$[1-14,3]$} & $0,0 \%$ & & 0,55 & $\square$ \\
\hline $\mathrm{HDA}^{* * * *}$ & $3,6 \%$ & {$[0,75-10,2]$} & $3,4 \%$ & {$[9,4-11,9]$} & $4,0 \%$ & {$[0,1-20,3]$} & 1 & $\square$ \\
\hline Obstrucción intestinal & $3,6 \%$ & {$[0,75-10,2]$} & $1,7 \%$ & {$[0-9,2]$} & $8,0 \%$ & {$[0,9-26]$} & 0,21 & $\square$ \\
\hline Anemia & $3,6 \%$ & {$[0,75-10,2]$} & $1,7 \%$ & {$[0-9,2]$} & $8,0 \%$ & {$[0,9-26]$} & 0,21 & $\square$ \\
\hline Otros & $44,8 \%$ & & & & & & & $\square$ \\
\hline Comorbilidad & & & & & & & & $\square$ \\
\hline $\mathrm{HTA}^{* * * * *}$ & $71,0 \%$ & {$[60-80,5]$} & $67,2 \%$ & {$[53,6-79]$} & $80,0 \%$ & {$[59,2-93,2]$} & 0,24 & $\square$ \\
\hline Enf respiratoria crónica & $28,9 \%$ & {$[19,5-39,9]$} & $32,7 \%$ & {$[21-46,3]$} & $20,0 \%$ & {$[6,8-40,7]$} & 0,24 & $\square$ \\
\hline DM $\|^{* * *}$ sin complicación & $25,0 \%$ & {$[16,5-36]$} & $27,6 \%$ & {$[16,6-40,9]$} & $20,0 \%$ & {$[6,8-40,7]$} & 0,47 & $\square$ \\
\hline Demencia & $16,8 \%$ & {$[9,5-26,7]$} & $15,5 \%$ & {$[7,3-27,4]$} & $20,0 \%$ & {$[6,8-40,7]$} & 0,75 & $\square$ \\
\hline Hipotiroidismo & $14,4 \%$ & {$[7,7-23,9]$} & $17,2 \%$ & {$[8,5-29,4]$} & $8,0 \%$ & {$[0,9-26]$} & 0,33 & $\square$ \\
\hline Insuficiencia cardiaca & $13,3 \%$ & {$[6,8-22,5]$} & $15,5 \%$ & {$[7,3-27,4]$} & $8,0 \%$ & {$[0,9-26]$} & 0,49 & $\square$ \\
\hline
\end{tabular}

*t-test; ${ }^{\top}$ MannWithey; $\square_{\chi 2}$. X: Mediana, *NAC: Neumonía adquirida en la comunidad, **ITU: Infección del tracto urinario,

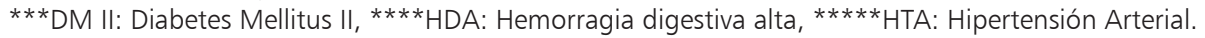

criterios de inclusión mencionados y que contaban con ficha clínica completa. La edad promedio fue de 79,4 años, 70,0\% corresponde a sexo femenino, la estadía hospitalaria fue 9,0 $\pm 5,6$ días promedio.

La mediana del consumo de fármacos previo al ingreso fue 4 medicamentos/persona, 6 durante la hospitalización y de 5 al egreso hospitalario.

Los pacientes presentaron una mediana de 3 comorbilidades, siendo la más prevalente hipertensión arterial con $71 \%$. En cuanto al índice de Charlson, la mediana fue de 2 puntos y un valor mayor de este índice se relacionó con un mayor consumo de fármacos, tanto al ingreso como al alta hospitalaria, con un índice de correlación de 0,25 y 0,33 respectivamente $(\mathrm{p}<0,05$ para ambas cifras). Las características restantes se encuentran en la Tabla 1. Los diagnósticos de ingreso más fre- cuentes fueron los de causa infecciosa (neumonía adquirida en la comunidad e infección del tracto urinario) con $28,9 \%$, no encontrándose diferencias estadísticamente significativas entre ambos sexos (Tabla 1).

En las características del manejo hospitalario destaca que $84,3 \%$ recibió apoyo kinesiológico, principalmente sexo femenino, encontrándose diferencias entre ambos géneros $(\mathrm{p}<0,005)$, $80,7 \%$ requirió apoyo de terapeutas ocupacionales y $59,0 \%$ apoyo psicológico, además $54,2 \%$ tuvo indicación de movilización, 9,6\% catéter urinario permanente y $8,4 \%$ sonda naso gástrica.

Al estudiar el índice de Barthel se encontró que la mediana de su valor basal fue de 90 puntos, 50 al ingreso y 80 al egreso ( $p<0,01)$. En $78,5 \%$ de los pacientes se presentó una disminución del IB 
Cambios en capacidad funcional en adultos mayores hospitalizados - V. Cares et al

Tabla 2. Evolución de la Capacidad Funcional

\begin{tabular}{|lccccccc|}
\hline & Total & RIC* & Femenino & RIC & Masculino & RIC \\
Índice Barthel & & & & & & \\
$\quad$ Basal & 90 & {$[65-95]$} & 85 & {$[65-95]$} & 90 & {$[65-100]$} & $\square$ \\
Ingreso & 50 & {$[30-75]$} & 52,5 & {$[40-80]$} & 45 & {$[10-70]$} & $\square$ \\
Egreso & 80 & {$[65-90]$} & 80 & {$[65-90]$} & 80 & {$[45-97,5]$} & $\square$ \\
Índice Lawton & & & & & & {$[2-6]$} & $\square$ \\
Basal & 4 & {$[2-7]$} & 4 & {$[2-7]$} & 4 & {$[0-3]$} & $\square$ \\
Ingreso & 2 & {$[0-4]$} & 3 & {$[0-5]$} & 1 & {$[2-6]$} & $\square$ \\
Egreso & 3 & {$[2-6]$} & 3 & {$[2-6]$} & 3 & {$[2]$}
\end{tabular}

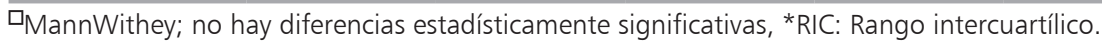

al ingreso respecto al basal con una mediana de 25 puntos. El $72,0 \%$ presentó un aumento del IB egreso respecto al ingreso con una mediana de 20 puntos. Finalmente, se encontró una relación estadísticamente significativa entre la diferencia del IB basal e ingreso y la diferencia entre el IB egreso e ingreso, con un índice de correlación de $-0,68,(\mathrm{p}<0,05)$.

El índice de Lawton basal presentó una mediana de 4 puntos, 2 al ingreso y 3 puntos al egreso $(\mathrm{p}<0,001)$, además existió una relación significativa entre la diferencia del IL basal e ingreso respecto al IL egreso e ingreso (Tabla 2).

La proporción de pacientes que presentaron delirium fue de 22,9\%, afectando al 19,0\% de las mujeres y al 32,0\% de los hombres sin diferencias significativas por género, con una edad promedio de 81,7 años.

Los pacientes con delirium presentaron un IB basal con una mediana de 65 y 18 al ingreso frente a un 90 y 53 puntos en aquellos que no lo presentaron, encontrándose diferencia estadísticamente significativa entre ellos. Aun cuando los valores del IB al egreso fueron menores en los pacientes con delirium, esta diferencia no fue significativa con respecto a los pacientes sin delirium. Por otro lado, estos últimos presentaron de forma estadísticamente significativa una menor estancia hospitalaria teniendo un promedio de 7,0 días, en comparación a 11,3 días en aquellos con delirium.

\section{Discusión}

La población estudiada presenta en su mayoría pluripatología, polifarmacia y en muchos casos han requerido más de una hospitalización en el año previo. El impacto que genera una enfermedad en el grupo de pacientes estudiados que requieren de hospitalización, traduce una pérdida de la capacidad funcional basal en $78,5 \%$ de los pacientes ingresados. De forma similar a otros estudios, el principal motivo de ingreso es la causa infecciosa, predominando las de origen respiratorio ${ }^{9,10}$. En cuanto a los días de hospitalización, se observó un promedio de 9,0 días, similar a lo encontrado en el estudio de Maña y cols ${ }^{7}$. De acuerdo a nuestros resultados, no fue posible establecer una relación significativa entre el ICh y días cama, es decir, aquellos pacientes con un puntaje más alto no presentaron estadías hospitalarias más prolongadas, lo que podría deberse a que este índice evalúa patologías crónicas, sin embargo, los principales motivos de ingreso al centro asistencial se debieron a patologías agudas, las que tienen resolución temprana al recibir los cuidados y tratamiento adecuado.

Considerando el IB obtenido, podemos destacar que el basal fue de 90 puntos lo que se traduce en un grado de dependencia leve en las ABVD, al momento de la hospitalización desciende a 50 presentando una dependencia moderada y finalmente al momento del alta se observó un IB de 80 volviendo a su estado basal. Comparando estos valores y los obtenidos en otros estudios que analizan a una población AM, podemos citar el de Formiga y cols ${ }^{10}$, el cual valora la capacidad funcional en 125 pacientes geriátricos de Barcelona, con un promedio de edad de 92 años y diversos motivos de ingreso: patologías médicas, quirúrgicas y traumatológicas, difiriendo esta población en estudio a la analizada en nuestro medio. No 
obstante, los pacientes presentaron una pérdida de su capacidad funcional basal de 33 puntos, cifra similar a lo observado en nuestros pacientes, con una mejoría de 43 puntos al egreso, cifra superior a la nuestra a pesar de la ostensible diferencia de edades entre ambos estudios. Sepúlveda y $\mathrm{cols}^{9}$ presentan a 93 pacientes ingresados a una UGA con una edad promedio de 92,8 años, los cuales recuperaban su IB basal en $48,0 \%$ al momento del egreso. En nuestro estudio esta variable alcanzó al 36,6\% de los pacientes, y $15,4 \%$ presentó una mejoría con respecto a su basal.

Estas diferencias podrían ser atribuibles a diversos factores, dentro de los cuales podemos destacar la experiencia de los sistemas geriátricos europeos adaptados a una población envejecida.

En la literatura existe evidencia que intervenciones interdisciplinarias (que incluyen incluso entrenamiento físico) están asociadas con una reducción del deterioro funcional ${ }^{16}$, lo que determina una disminución de la estadía hospitalaria ${ }^{16,17}$, menor readmisión hospitalaria y uso de casas de reposo ${ }^{17,18}$, reducción en la incidencia de caídas $^{17-20}$ y mejoría en la percepción del estado de salud ${ }^{21}$. La evidencia muestra que estos efectos están presentes entre 6 a 12 meses después del inicio de la intervención interdisciplinaria con el mayor efecto a los 3 meses $^{22}$.

En cuanto al delirium podemos destacar el estudio de González-Colaço y $\operatorname{cols}^{23}$, en el cual se presenta una prevalencia de $20,7 \%$, una edad promedio de 85,3 años, un ICh de 2,9 puntos, donde se observaron diferencias estadísticamente significativas en el IB basal, IB ingreso y estancia hospitalaria en comparación a pacientes sin delirium, obteniéndose resultados similares en nuestro estudio. Esto comprueba la alta frecuencia de este trastorno en los AM, sus repercusiones médicas y socioeconómicas, por lo tanto, es imperante el diagnóstico precoz y un manejo interdisciplinario adecuado.

Una de las limitaciones encontradas es que al utilizar fichas clínicas como fuente de información, la medición de los índices de funcionalidad (IB e IL) se realizaron al egreso hospitalario, sin considerar la evolución que pudieran tener tras el alta. Es preciso considerar que la descripción del servicio de geriatría no representa necesariamente a todos los AM hospitalizados en una UGA u otros servicios de medicina, ya que los criterios de inclusión y exclusión seleccionaron sólo al 22\% de los pacientes. La principal causa fue la exclusión de pacientes derivados desde otras unidades hospitalarias, con el objetivo de aislar la injerencia que tiene la UGA en la evolución de su capacidad funcional. Por lo tanto, a futuro sería interesante realizar estudios comparativos con pacientes derivados desde otras unidades y con otros servicios de Medicina con el fin de determinar las diferencias y los beneficios que implica la hospitalización en una UGA.

Nuestro estudio muestra, al igual que la literatura internacional, que la evaluación geriátrica integral aplicada en unidades especializadas a través del trabajo interdisciplinario de geriatras, enfermeras, kinesiólogos, terapeutas ocupacionales, farmacéuticos clínicos y psicólogos tienen un impacto positivo en la evolución de la capacidad funcional de los AM durante su hospitalización permitiendo una mayor independencia a su alta médica lo que se asocia a una mejor calidad de vida $^{17-19,21}$, además una reciente revisión mostró una mayor sobrevida, probabilidad de regresar al hogar post hospitalización y mejor funcionamiento cognitivo en AM atendidos en una unidad geriátrica, beneficios suficientes para justificar la reorganización de los servicios que atienden a AM, resultando desde un punto de vista social en una potencial reducción de $\operatorname{costos}^{24}$, por lo que sería aconsejable implementar este enfoque de trabajo en la atención de todo AM atendido en los centros asistenciales del país.

A partir de los resultados obtenidos, las experiencias en otros países y la evidencia existente, nos parece imperativa una mayor presencia de médicos especializados en AM junto con la implementación y masificación de estos servicios, tanto en la atención pública como en la privada, considerando el envejecimiento poblacional progresivo y los costos socio económicos que esto implica. 


\section{Anexos. Instrumentos utilizados}

\section{Anexo 1. Versión original Índice de comorbilidad de Charlson}

\section{Parámetro}

Infarto de miocardio: debe existir evidencia en la historia clínica que el paciente fue hospitalizado por ello, o bien evidencias de que existieron cambios e enzimas y/o en ECG

Insuficiencia cardiaca: debe existir historia de disnea de esfuerzos y/o signos de insuficiencia cardiaca en la exploración física que respondieron favorablemente al tratamiento con digital, diuréticos o vasodilatadores. Los pacientes que estén tomando estos tratamientos pero no podamos constatar que hubo mejoría clínica de los síntomas y/o signos, no se incluirán como tales

Enfermedad arterial periférica: incluye claudicación intermitente, interveidos de by-pass arterial periférico, isquemia arterial aguda y aquellos con aneurisma de la aorta (torácica o abdominal) de $>6 \mathrm{~cm}$ de diámetro

Enfermedad cerebrovascular: paciente con AVC con mínimas secuelas o AVC transitorio

Demencia: pacientes con evidencia en la historia clínica de deterioro cognitivo crónico

Enfermedad respiratoria crónica: debe existir evidencia en la historia clínica, en la exploración física y en exploración 1 complementaria de cualquier efermedad respiratoriao crónica, incluyendo EPOC y asma

Enfermedad del tejido conectivo: incluye lupus, polimiositis, enfermedad mixta, polimialgia reumática, arteritis de celulas gigantes y artritis reumatoide

Úlcera gastroduodenal: incluye a aquellos que han recibido tratamiento por un ulcus y quellos que tuvieron sangrado por úlceras

Hepatopatía crónica leve: sin evidencias de hipertensión portal, incluye pacientes con hepatitis crónica

Diabetes: incluye los tratados con insulina o hipoglicemiantes, pero sin complicaciones tardías, no se incluirán los tratados únicamente con dieta

Hemiplejia: evidencia de hemiplejia o paraplejia como consecuencia de un AVC u otra condición

Insuficiencia renal crónica moderada/severa: incluye pacientes en diálisis, o bien con creatininas $>3 \mathrm{mg} / \mathrm{dl}$ objetivadas de forma repetida y mantenida

Diabetes con lesión en órganos diana: evidencia de retinopatía, neuropatía o nefropatía, se incluyen también antecedentes de cetoacidosis o descompensación hiperosmolar

Tumor o neoplasia sólida: incluye pacientes con cáncer, pero si metástasis documentadas

Leucemia: incluye leucemia mieloide crónica, leucemia crónica, policitemia vera, otras leucemias crónicas y todas las leucemias agudas

Linfoma: incluye todos los linfomas, waldestrom y mieloma

Hepatopatía crónica moderada/severa: con evidencia de hipertensión portal (ascitis, várices esofágicas o encefalopatía)

Tumor o neoplasia sólida con metástasis 


\section{Anexo 3. Índice de Lawton \& Brody (actividades instrumentales de la vida diaria)}

\begin{tabular}{|rr} 
Parámetro & Puntuaci \\
Uso teléfono & 1 \\
Utilizar el teléfono por propia iniciativa y marcar los números & 1 \\
Sabe marcar números conocidos & 1 \\
Contesta al teléfono, pero no sabe marcar & 0 \\
No utiliza el teléfono en absoluto & 0
\end{tabular}

\section{Compras}

Realiza todas las compras necesarias de manera independiente 1

Sólo sabe hacer pequeñas compras 0

Ha de ir acompañado para cualquier compra 0

Completamente incapaz de hacer la compra 0

\section{Preparación comida}

Organiza, prepara y sirve las comidas por sí solo adecuadamente $\quad 1$

Prepara adecuadamente las comidas si se le proporcionan los ingredientes $\quad 0$

Prepara, calienta y sirve las comidas, pero no sigue una dieta adecuada $\quad 0$

Necesita que le preparen y sirvan las comidas 0

\section{Cuidado de la casa}

Mantiene la casa solo o con ayuda ocasional (para trabajos pesados) 1

Realiza tareas ligeras, como lavar los platos o hacer las camas 1

Realiza tareas ligeras, pero no puede mantener un adecuado nivel de limpieza $\quad 1$

Necesita ayuda en todas las labores de la casa $\quad 1$

No participa en ninguna labor de la casa $\quad 0$

\section{Lavado de la ropa}

Lava por sí solo toda su ropa $\quad 1$

Lava por sí solo pequeñas prendas $\quad 1$

Todo el lavado de ropa debe ser realizado por otro 0

\section{Uso de medios de transporte}

Viaja solo en transporte público o conduce su propio coche 1

Es capaz de coger un taxi, pero no usa otro medio de transporte $\quad 1$

Viaja en transporte público cuando va acompañado por otra persona 1

Sólo utiliza el taxi o el automóvil con ayuda de otros 0

No viaja

\section{Medicamentos}

Es capaz de tomar su medicación a la hora y con la dosis correcta $\quad 1$

Toma su medicación si la dosis le es preparada previamente 0

No es capaz de administrarse su medicación $\quad 0$

\section{Manejo asuntos económicos}

Se encarga de sus asuntos económicos por sí solo 1

Realiza las compras de cada día, pero necesita ayuda en las grandes compras, bancos 1

Incapaz de manejar dinero

La máxima dependencia estaría marcada por la obtención de 0 puntos, mientras que una suma de 8 puntos expresaría una independencia total. 
Anexo 2. Índice de Barthel (versión modificada de Ganger et al.) (Actividades básicas de la vida diaria)

\begin{tabular}{|c|c|c|c|}
\hline Parámetro & Sin ayuda & Con ayuda & No realiza \\
\hline Beber de un vaso & 4 & 0 & 0 \\
\hline Comer & 6 & 0 & 0 \\
\hline Vestirse parte superior del cuerpo & 5 & 3 & 0 \\
\hline Vestirse parte inferior del cuerpo & 7 & 4 & 0 \\
\hline Ponerse órtesis o prótesis & 0 & 2 & No aplicable \\
\hline Actividades de aseo & 5 & 0 & 0 \\
\hline Lavarse o bañarse & 6 & 0 & 0 \\
\hline Control de orina & 10 & 5 accidental & 0 \\
\hline Control intestinal & 10 & 5 accidental & 0 \\
\hline Sentarse/levantarse cama/silla & 15 & 7 & 0 \\
\hline Utilización de WC & 6 & 3 & 0 \\
\hline Entrar/salir bañera o ducha & 1 & 0 & 0 \\
\hline Caminar 50 m en Ilano & 15 & 10 & 0 \\
\hline Subir/bajar trozo de escalera & 10 & 5 & 0 \\
\hline Si no camina, impulsa sillas ruedas & 5 & 0 & No aplicable \\
\hline
\end{tabular}

Puntuación total: 0-100. Dependencia leve: 61-99. Dependencia moderada: 41-60. Dependencia severa: 21-40. Dependencia total: $0-20$.

\section{Anexo 4. Detección del síndrome confusional agudo mediante algoritmo Confusión Assesstment Method} (CAM)

Criterio 1: Comienzo agudo y fluctuante

Hay evidencia de un cambio agudo o reciente en el estado mental del paciente, o bien la conducta o el estado mental fluctúa durante el día

\section{Criterio 2: Desatención}

El paciente tiene dificultad para concentrarse, se distrae fácilmente

\section{Criterio 3: Pensamiento desorganizado}

El paciente tiene una conversación vaga, incoherente, ilógica, flujo de ideas o cambios no justificados de un tema a otro

\section{Criterio 4: Nivel de conciencia disminuido}

La respuesta a preguntas es lenta, o bien está somnoliento

Para establecer la sospecha de síndrome confusional (delirium) se requiere los criterios 1 y 2 de forma obligada y uno cualquiera de los criterios 3 y 4

\section{Referencias}

1. Centro Latinoamericano y Caribeño de Demografía (CELADE)-División de Población Comisión Económica para América Latina y el Caribe Santiago de Chile. Los Adultos Mayores en América Latina y el Caribe Datos e Indicadores. Boletín Informativo. Marzo de 2002.

2. Marín P. Reflexiones para considerar en una política pública de salud para las personas mayores. Rev Med Chile 2007; 135: 392-8.

3. Baztán JJ, Suárez-García FM, López-Arrieta J, Rodríguez-Mañas L, Rodríguez-Artalejo F. Effectiveness of acute geriatric units on functional decline, living at home, and case fatality among older patients admitted to hospital for acute medical disorders: meta-analysis. BMJ 2009; 338: b50. 
4. Bakker TJ, Duivenvoorden HJ, van der Lee J, Krulder JW, Driesen JJ, Ribbe MW. Prevalence and prognostic importance of riskfactors for long hospital stay within elderly patients admitted to a hospital; a clinical-empirical study. Tijdschrift Voor Gerontologie En Geriatrie 2010; 41 (4): 177-86.

5. Gutiérrez J, Domínguez V, Solano JJ. [Functional deterioration secondary to hospitalization for an acute disease in the elderly. An analysis of its incidence and the associated risk factors]. Rev Clin Esp 1999; 199: 418-23.

6. Covinsky KE, Palmer RM, Fortinsky RH, Counsell SR, Stewart AL, Kresevic D. Loss of independence in activities of daily living in older adults hospitalized with medical illnesses: Increased vulnerability with age. J Am Geriatr Soc 2003; 51 (4): 451-8.

7. Mañas MD, Marchán E, Conde C, Sánchez S, SánchezMaroto T, Molina MC. [Functional impairment in elderly patients hospitalized in an Internal Medicine unit]. An Med Interna 2005; 22 (3): 130-2.

8. Sager MA, Rudberg MA, Jalaluddin M, Franke T, Inouyse SK, Landefeld CS, Siebens H, Winograd CH. Hospital admission risk profile (HARP): identifying older patients at risk for functional decline following acute medical illness and hospitalization. J Am Geriatr Soc 1996; 44 (3): 251-7.

9. Sepúlveda D, Isaac M, Izquierdo G, Ruiperez I. Deterioro funcional en pacientes nonagenarios ingresados en hospitales de agudos. Med Clin (Barc) 2001; 116: 799.

10. Formiga F, López A, Sacanella E, Jacob X, Masanes F, Vidal M. Valoración de la capacidad funcional después de un ingreso hospitalario en pacientes nonagenarios. Med Clin (Barc) 2000; 115: 695-6.

11. Mahoney FI, Barthel DW. Functional evaluation: Barthel index. Md State Med J 1965; 14: 61-5.

12. Pfeiffer E. A short portable mental status questionnaire for the assessment of organic brain deficits in the elderly. J Am Geriatr Soc 1975; 23: 433-41.

13. Charlson M, Pompei P, Ales KL, McKenzie CR. A new method of classyfing prognostic comorbidity in longitudinal studies: development and validation. J Chron Dis 1987; 40: 373-83.

14. Lawton MP, Brody EM. Assessment of older people: selfmaintaining and instrumental activities of daily living. Gerontologist 1969; 9: 179-86.

15. Inouye SK, Van Dyck CH, Alessi CA, Balkin S, Siegal AP, Howitz, RI. Clarifying confusion: the confusion assessment method. A new method for detection of delirium. Ann Intern Med 1990; 113: 941-8.

16. De Morton NA, Keating JL, Jeffs K. The effect of exercise on outcomes for older acute medical inpatients compared with control or alternative treatments: A systematic review of randomized controlled trials. Clinical Rehabilitation 2007, 21 (1): 3-16.

17. Beswick AD, Rees K, Dieppe P, Ayis S, Gooberman-Hill $\mathrm{R}$, Horwood J, et al. Complex interventions to improve physical function and maintain independent living in elderly people: A systematic review and meta-analysis. Lancet 2008, 371 (9614): 725-35.

18. Baztán JJ, Suárez-García FM, López-Arrieta J, Rodríguez-Manas L, Rodríguez-Artalejo F. Effectiveness of acute geriatric units on functional decline, living at home, and case fatality among older patients admitted to hospital for acute medical disorders: Metaanalysis. British Medical Journal 2009; 338: b50.

19. Van Craen K, Braes T, Wellens N, Denhaerynck K, Flamaing J, Moons $\mathrm{P}$, et al. The effectiveness of inpatient geriatric evaluation and management units: A systematic review and meta-analysis. J Am Geriatr Soc 2010; 58 (1): 83-92. Epub 2009 Dec 9.

20. Gillespie LD, Gillespie WJ, Robertson MC, Lamb SE, Cumming RG, Rowe BH. Interventions for preventing falls in elderly people. Cochrane Database of Systematic Reviews 2003, Issue 4. Art. No.: CD000340.

21. Caplan GA, Williams AJ, Daly B, Abraham K. A randomized, controlled trial of comprehensive geriatric assessment and multidisciplinary intervention after discharge of elderly from the emergency department? the DEED II study. J Am Geriatr Soc 2004; 52 (9): 141723.

22. Cunliffe AL, Gladman JR, Husbands SL, Miller P, Dewey ME, Harwood RH: Sooner and healthier: A randomised controlled trial and interview study of an early discharge rehabilitation service for older people. Age and Ageing 2004; 33 (3): 246-52.

23. González-Colaço Harmand M, Medina Domínguez L, Hornillos Calvo M. Prevalencia de delírium al ingreso en una unidad de agudos de geriatría. Med Clin (Barc) 2008; 131: 117.

24. Ellis G, Whitehead MA, O'Neill D, Langhorne P, Robinson D. Comprehensive geriatric assessment for older adults admitted to hospital. Cochrane Database Syst Rev 2011; (7): CD006211. 